Voix et Images

voixetimages

\title{
Langage poétique : écart ou errance du sens
}

\section{Noël Audet}

Volume 3, numéro 3, avril 1978

Pierre Perrault

URI : https://id.erudit.org/iderudit/200124ar

DOI : https://doi.org/10.7202/200124ar

Aller au sommaire du numéro

Éditeur(s)

Les Presses de l'Université du Québec

ISSN

0318-9201 (imprimé)

1705-933X (numérique)

Découvrir la revue

Citer cet article

Audet, N. (1978). Langage poétique : écart ou errance du sens. Voix et Images, 3(3), 459-466. https://doi.org/10.7202/200124ar d'utilisation que vous pouvez consulter en ligne.

https://apropos.erudit.org/fr/usagers/politique-dutilisation/ 


\section{Langage poétique: écart ou errance du sens}

Livre trop ou trop peu critiqué depuis sa parution en 1966, Structure du langage poétique de Jean Cohen formulait d'une façon plus scientifique une série de problèmes "poétiques" que la rhétorique traditionnelle s'était contentée de répertorier.

Toutefois, même si plusieurs clarifications semblent justes en ce qui a trait au fonctionnement spécifique du langage poétique, la notion même d'écart, centrale dans la théorie de Cohen, ne peut pas être retenue pour diverses raisons.

Examinons brièvement le fondement méthodologique de cette notion.

Il s'agit pour nous de confronter le poème avec la prose. Et puisque la prose est le langage courant, on peut le prendre pour norme et considérer le poème comme un écart par rapport à elle 1.

On voit donc comment, dès le départ, la prose est prise comme langage "naturel ", étalon de mesure, et comment le style ne peut être qu'une déviation par rapport à cette norme. Et de fil en aiguille, l'auteur sera amené à poser deux types de fonctionnement dans le langage poétique: d'abord la position de l'écart et dont la lecture sera perçue comme impertinente; ensuite la réduction de l'écart, dans un second temps, soit le rétablissement de la vraie pertinence, métaphorique celle-là.

La stratégie poétique a pour seule fin le changement de sens. Le poète agit sur le message pour changer la langue. Si le détour est nécessaire, c'est parce que le chemin direct qui va de $\mathrm{Sa}$ à Sér est barré. Entre les deux s'interpose Sé, qu'il faut écarter en un premier temps, pour qu'en un second temps Séz prenne sa place. Si le poème viole le code de la parole, c'est pour que la langue le rétablisse en se transformant ${ }^{2}$.

Autant dire que l'écriture poétique consisterait à reprendre à la prose le plan du contenu dont il suffirait d'écarter le plan d'expression, autrement dit le poète se contenterait d'ajouter des ornements rhétoriques à un discours idéologique préexistant, ce qui aurait pour effet de transfor- 
mer en retour ce premier niveau de sens, et le poète en arriverait ainsi à «changer la langue». Si la perception des divers éléments en cause paraît juste, leur fonctionnement soulève trop d'obstacles théoriques pour prétendre rendre compte du phénomène.

- Le discours poétique est aussi premier que le discours de prose, et bien qu'il soit difficile de le prouver, il semble même qu'il soit premier tout court, historiquement et sur le plan psychique. Dans l'histoire de l'humanité, les découvertes des ethnologues nous incitent à penser que le langage des civilisations primitives était métaphorique, c'est-à-dire qu'il exprimait d'abord des relations d'ordre symbolique ou mythique. Dans cette perspective, ce qu'on appelle la prose ne serait que le produit d'une censure culturelle grâce au développement de l'abstraction et de la maîtrise technologique de l'homme sur le monde. Quant au plan psychique, dans la synchronie du prócessus actuel d'écriture, il ne me paraît pas plus évident que le sens "prosaïque" préexiste au sens poétique qui se substituerait à lui. C'est ce que je vais tenter de montrer dans les lignes qui suivent.

Jean Cohen affirme en effet que le sens dénotatif doit être bloqué pour que le sens connotatif se manifeste et en conséquence que la signification globale du poème fonctionne:

La signification émotionnelle est antithétique de la signification intellectuelle, et il faut en conséquence nécessairement "faire obstacle" à celle-ci pour assurer le triomphe de celle-là ${ }^{3}$.

Or il me semble précisément tout au contraire que la signification poétique n'est possible que parce que les deux niveaux fonctionnent simultanément, sinon l'opération de lecture consisterait à traduire littéralement en un langage second ce qui était voilé dans son expression première (le texte), et mieux vaudrait joindre au texte son mode d'emploi, lexique inclus, ou réécrire le poème.

Prenons l'exemple avancé par Jean Cohen lui-même, soit les “bleus angélus" de Mallarmé. II nous dit en substance qu'un angélus (auditif) bleu (visuel) n'a pas de sens - signification impertinente du niveau l écartée au profit d'une signification II (connotée): angélus tendres, clairs, désespérants, etc. Le sens a viré de façon irréversible sur le mot «bleus"qui se trouve aboli du fait même. Et la seconde lecture réécrirait la signification du poème sans s'empêtrer dans le piège des mots, s'il faut pousser la théorie dans ses derniers retranchements. Et l'on revient à l'inutile ornement rhétorique qui masquerait le sens.

Tout autrement, il m'apparaît que le sens connoté n'est pensable et possible que dans sa relation étroite avec le fonctionnement du syntagme dans son sens propre et dont il est contemporain. La signification " poétique" ne s'écarte pas d'un sens rendu impertinent en le bloquant pour enfin l'évacuer, au contraire c'est par la faille de cette expression même que la signification jaillit et erre d'un niveau de signification à l'autre. Cette errance procède d'ailleurs d'une façon dialectique: le sens surgit du syn- 
tagme comme de sa base propre (parole liée) qui en règle la fermeture tout en lui imprimant une direction vers le paradigme; le sens s'ouvre dans le paradigme (parole libérée) pour revenir se heurter au sens apparent du syntagme et ainsi de suite.

Si Grandbois écrit "ton œil comme un feuillage" (ce qui ne veut pas dire avoir une paille dans l'œil), il y a bien intersection phonologique (entre feuill- et œil) et intersection de champs sémiques entre feuillage au sens propre et un certain vaporeux du regard, par exemple, ou la qualité ou le mouvement des cils ou le mouillé de l'œil, etc. Et l'errance du sens n'est possible - et c'est même là le seul lieu ou l'espace de la poésie que parce que les niveaux du sens sont tous maintenus à la fois dans une tension dialectique. Quand Gaston Miron écrit: “Quel cœur effaré de chevreuse en fuite" (l'Homme rapaillé, p. 52), matériellement, linguistiquement, il dit bien ce que ça dit, mais il dit plus aussi en ce sens qu'il dit plus que ce qu'il dirait s'il décidait d'en extraire un sens et de l'exprimer directement. C'est pourquoi il écrit ce qu'il veut dire mais sans le dire, il l'écrit d'ailleurs trois fois plutôt qu'une. J'y reviendrai. Imaginons maintenant un tableau illustrant le travail de la signification; on y trouvera $S$ comme dans Scène, Signifiance, Syntagme - et $\mathbf{P}$ comme dans Parole déliée, Production de la signification, Paradigme:

\section{TABLEAU 1}

(S)

(P)

$\begin{array}{ll}\text { cils } & \text { feuilles } \\ \text { fragilité } & \text { ramilles } \\ \text { mouvement } & \text { frêles } \\ \text { yeux verts } & \text { en mouvement } \\ \text { mouillés } & \text { vertes } \\ \text { regard } & \text { mouillées } \\ \text { lumière } & \text { reflets } \\ \ldots & \ldots\end{array}$

«ton œil comme un feuillage»

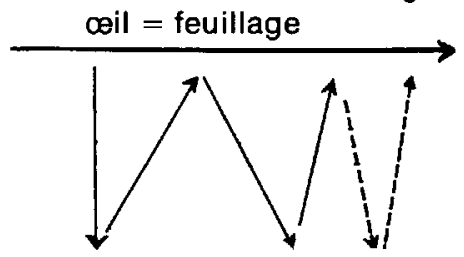

ton œil/comme un feuillage

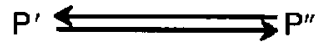

(sens de surface)

+ indice

ERRANCE

(sens potentiel)

Comme j'ai déjà cru le démontrer dans des analyses antérieures de Saint-Denys Garneau et d'Alain Grandbois, le phénomène poétique semble bien consister surtout dans la réversibilité des deux axes du lan- 
gage. D'autre part, si le syntagme tend à rétrécir le sens, à l'endiguer de plus en plus en raison de son développement même, la poésie a pour effet de renverser les termes dans leur appartenance paradigmatique. Or si le niveau $P$ (paradigmatique) porte une signification repérable, c'est bien parce qu'en $S$ le terme "feuillage" signifie feuillage, de même que le terme «ill" prend aussi son sens propre. C'est dire que le sens dénoté (selon la théorie de Cohen), loin d'être aboli, continue d'agir positivement et préside sans relâche au va-et-vient du sens entre $S, P, P 1$, et $P^{\prime \prime}$. On pourrait faire la même démonstration avec les "bleus angélus". Du signifiant lié dans et par le syntagme au signifiant libéré dans le paradigme au signifiant lié, etc. On peut même dire que l'origine de la signification gît dans le lié des signifiants, et que c'est la forme du lien unissant les signifiants entre eux (avec leurs significations propres) qui induit et détermine l'errance du sens, en pose la direction en même temps que la limite. La rhétorique s'est attaquée à ce problème et a relevé les différentes formes de liaisons avec leurs contraintes et leurs effets de sens.

En conclusion à ce problème, on constate qu'il ne saurait y avoir substitution du niveau $P$ au niveau $S$ sous peine de tomber dans le nonsens, le non-langage, c'est-à-dire de retourner à la nomenclature du dictionnaire où les mots signifient tout et rien hors le syntagme qui les lie pour en fermer l'ouverture.

\section{Les indices du sens}

Cependant, si l'on conçoit relativement bien comment le lecteur passe d'un niveau à l'autre dans un mouvement dialectique, on n'est pas plus avancé quant à savoir pourquoi il le fait, alors que la lecture d'un texte journalistique par exemple ne requiert pas le même battement psychologique - ou si elle y recourt, c'est pour effacer à mesure les faux sens ou les sens non pertinents du paradigme (entreprise de censure) et réduire la signification en la précisant de plus en plus au déroulé du syntagme.

Concédons à Jean Cohen que le passage d'un niveau à l'autre fait problème. Pour le résoudre, je suggère la solution suivante: se trouvent inscrits dans notre niveau $S$, en même temps qu'un sens, un indice que ce sens n'est pas le seul, indice donc d'un autre fonctionnement. En l'absence de tels indices, ou aiguilleurs du sens, l'autre niveau de signification ne serait pas lisible, ou à tout le moins le lecteur pourrait s'en passer - ce qui n'est pas le cas. Et lorsqu'un lecteur dit ne pas «comprendre» la poésie, il me semble après diverses expériences, que c'est précisément parce qu'il ne lit pas les indices qui le conduiraient au niveau $P$ et l'en ramèneraient. Ces indices auraient à mon avis deux formes contraires qui pourraient procéder en dernier ressort du même principe: l'évidement (the gap) ou le creux produit soit dans le signifiant, soit dans la chaîne signifiante ellemême. 


\section{Indices du manque}

D'une part, il s'agit d'un manque, d'une fissure syntaxique ou sémantique qui appelle le plein, en d'autres termes c'est l'appel d'air d'une forme vide. Contrairement à ce que pensent beaucoup de gens, écrire un poème, ce n'est pas enrichir une parole jusqu'à ce qu'ellè déborde d'un supplément de significations. La poésie est plutôt la mise en place d'un système signifiant dont la stratégie a pour effet d'instaurer le creux en chaque mot de manière que le plus grand nombre de significations possible accourent au moment où la lecture s'en empare et transforme le texte en discours (ou fait passer de la signifiance à la signification). Le poème est donc un appareil évidé, troué de part en part, dont les structures nues se renvoient les reflets. Ce système de signifiants devient relativement autonome par rapport à la masse flottante des signifiés. Loin d'être «une violation systématique des règles du langage (Cohen, p. 133-134), le langage poétique c'est le code de la langue qui fonctionne à nu et comme à vide, décroché partiellement du plan du contenu; la poésie tire ses effets de sens d'une surutilisation du code de la langue, c'est-à-dire qu'elle multiplie les rapports formels des éléments linguistiques entre eux, au lieu de chercher à modifier ces rapports au nom d'un soi-disant contenu préalable. Mitsou Ronat s'insurge également contre l'hypothèse du viol de la langue:

la poésie ne viole jamais les lois de la langue, [...] ne sort jamais de la langue. Tout au plus, elle peut déplacer, à la manière des schizophrènes, le champ d'application d'une règle existante ${ }^{4}$.

Pour expliquer cette procédure d'évidement selon laquelle les mots se creusent pour ne conserver que leur forme ou leur contour (idée qui m'était bizarrement venue à la lecture d'Althusser en 1972) je ne trouve pas aujourd'hui de meilleure explication qu'en me reportant à la définition que donne Mitsou Ronat du nom propre:

le nom propre est précisément un signifiant pur, sans signifié autre que le référent, ou plutôt c'est un index dont le seul signifié est la désignation, la désignation du référent. C'est le code renvoyant au code ${ }^{5}$.

D'une manière analogue; le mot en poésie serait un index, fonctionnant comme un nom propre dont le référent n'est pas une personne ou un objet unique mais bien le paradigme. C'est-à-dire que chaque signifiant en poésie est le nom propre d'un paradigme. Cette idée séduisante appelle toutefois quelques précisions. Si elle explique en effet l'ouverture du mot, ou ce que je nomme terme délié dans le paradigme - c'est le «mot en liberté" des Surréalistes - elle ne rend pas compte de la fermeture relative du sens ou comment échapper à un discours qui signifierait n'importe quoi. Or les significations poétiques sont tout de même précises, bien que polyvalentes, et ce n'est pas parce qu'elles contiennent une part de prose ( "Le message poétique est à la fois vers et prose", Cohen, p. 101.) mais bien parce que le syntagme enchaîne les signifiants l'un à l'autre et que/ 
c'est cette forme syntaxique qui détermine de façon contraignante la modalité de leur interrelation (la métaphore ne travaille pas la langue comme la métonymie, etc.). En d'autres termes, S, la Scène avec son discours de surface relaie constamment l'errance et sert à tisser et à soutenir comme sa trame chacun des autres niveaux de la signification. Ces derniers sont relayés, c'est-à-dire produits et orientés par la structure syntagmatique, et l'on pourrait dire que la poésie est pure fonction d'aiguillage du sens, production des indices du sens à partir d'un sens de surface déçu, maintenu entre parenthèses pour servir essentiellement de relais. Le signifiant en poésie ne fonctionne donc pas purement et simplement comme le nom propre d'un paradigme: s'y trouve, en plus de cet index pointé en direction du paradigme, une signification de surface, bien présente mais suspendue alternativement.

Nous avons donc d'une part les indices constitués par un manque, une déception, une rupture soit dans la continuité logico-sémantique, soit dans le rythme ou la matérialité du signifiant lui-même. Les indices dont nous parlons recoupent les figures et tropes de la rhétorique traditionnelle mais pourraient théoriquement s'étendre au-delà.

Ainsi quand Miron écrit "ma terre amère ma terre amande 6 ", ce qui nous retient avant même le sens du mot camande» (niveau $S$ ) et ses connotations (niveau $P$ ), c'est le manque dans la répétition amorcée puis déçue dans sa dernière syllabe (-mère/-mande) et qui ordonne l'autre lecture.

\section{Indices du trop-plein (signifiant)}

D'autre part, le texte poétique fonctionne au trop-plein de signifiants (qui n'est qu'un corollaire du manque dans le signifié), ce qui revient à produire le même effet. Soit le vers de Miron: "Quel cœur effaré de chevreuse en fuite ${ }^{7}$. Où est l'écart, de quoi à quoi? II n'y en a pas dans le vers lui-même, mais il y a une formidable errance vers laquelle nous aiguille l'indice du trop-plein. C'est la surabondance des signifiants, avec allitérations qui supportent autant de redondances sémantiques. Voilà pourquoi je disais plus haut qu'un poète ne dit pas ce qu'il veut dire mais qu'il l'écrit trois fois plutôt qu'une. En effet, Miron ne s'est pas contenté de dire: pourquoi t'enfuis-tu, ou me fuis-tu? car le poème n'aurait pas eu liẹu (le lieu) de s'écrire. Si c'est bien là ce qu'il veut dire, il ne le dit pas pour mieux le signifier à travers un système de signifiance qui construit l'errance et dit finalement beaucoup plus que cela, tout en traversant quatre fois cette même signification dans un seul vers. Voici comment. 
TABLEAU ॥

L (Pourquoi me fuis-tu?)

$S$ quel coeur effaré / de chevr/euse / en fuite

$\mathbf{P} \quad \mathbf{P}^{\prime} \quad \mathbf{P}^{\prime \prime} \quad \mathbf{P}^{\prime \prime \prime} \quad \mathbf{P}^{\prime \prime \prime \prime}$

où $\mathrm{P}^{\prime}=$ effroi, stupeur, amour sur le point de fuir, etc.

$P^{\prime \prime}=$ chevreuil, innocence de l'animal doux mais peureux, seul comportement caractérisé devant l'homme: la fuite.

$\mathrm{P}^{\prime \prime \prime}=$ chevreuse : forme féminine, néologisme instable dans sa terminaison, forme fuyante...

$P^{\prime \prime \prime \prime}=$ en fuite, fuite en train de se réaliser.

C'est parce que ces mots s'ajoutent sans nécessité logique apparente qu'ils indiquent par le fait même l'autre nécessité qui les gouverne. Miron a écrit quatre fois ce qu'il voulait dire, mais il n'a pas dit quatre fois: tu me fuis, parce que tu as peur et t'enfuis, même dans ton langage qui fuit, parce que tu es en fuite. C'est bien pourtant là la logique du discours de surface qui permet à la signification de rebondir et de s'enrichir à chaque traversée d'un nouveau paradigme. Et cela n'est possible que parce que les signifiants s'évident au fur et à mesure que le creux ainsi produit se trouve aussitôt surcomblé par les mécanismes linguistiques eux-mêmes (l'irruption du paradigmatique) et d'autre part la mythique et les fantasmes individuels.

Si j'appelle $L$ le niveau logique ou une réduction idéologique du sens - qui n'existe nulle part sous cette forme et dont il conviendra de préciser le statut - il devient possible de construire la formule suivante: fier SPnS $\stackrel{(!)}{(!)}]$

[le poète ne dit pas $L$ pour écrire-construire $S$ afin de mieux signi-

II ne serait pas impensable que Verlaine ait mijoté quelque chose du genre en écrivant: "Ne va pas choisir tes mots sans quelque méprise", la méprise constituant précisément l'indice de l'autre fonctionnement cependant que le même signifiant pose une première strate du sens.

Soit. Le discours poétique établit des rapports pleins entre deux contours d'absence, et ce travail du langage est lisible grâce aux indices résultant d'un manque ou d'un trop-plein. Mais qu'arrive-t-il lorsque le syntagme, le vers ou même le poème ne contient à peu près aucun indice des autres sens? Cette condition est-elle suffisante pour exclure le texte du genre dit poétique?

Revenons à un vers de Miron qui s'écrit: “Jamais je n'ai fermé les yeux ${ }^{8}$. En l'absence du contexte de la page en question, on remarque qu'il n'y a là aucune distorsion linguistique; le sens dénoté fonctionne, on pourrait tout au plus convaincre Miron d'exagération ou de mensonge. Dans l'hypothèse où tout le texte aurait la même tenue, il faudrait faire intervenir l'institution littéraire qui seule est en mesure d'imposer une lecture "poétiquen - ce que je tenterai de préciser dans un prochain article. 
Pour mes fins présentes, il suffit de replacer le vers dans son contexte pour constater qu'il fonctionne comme les autres. L'indice du trop-plein se manifeste ici sous la forme du "jamais", et le reste de la strophe ne fera que déplier le paradigme de la volonté de conscience sans faille. C'est dire que même ici, les signifiants fonctionnent aussi comme les noms propres du paradigme et que la signification va se construire en face de cette phrase, ou dans l'espace produit qui constitue l'architrope de toute poésie: la mise en creux. Le référent du syntagme "fermé les yeux" est en effet le paradigme de ce qui fait toujours fermer les yeux: vertige / volupté / tendresse / euphorie / proximité du feu (chaleur) / ou de la lumière trop vive / fatigue / sommeil - dont la négation absolue, en l'absence d'autres figures, constituait l'indice.

Jamais je n'ai fermé les yeux

malgré les vertiges sucrés des euphories

même quand mes yeux sentaient le roussi

même en butte aux rafales montantes du sommeil ${ }^{9}$

Pour résumer ce que je crois acquis, le langage poétique ne se compose pas d'écarts relativement à un discours conçu comme premier; cependant il organise ou construit un système d'errance du sens et c'est par là qu'il produit un réseau de significations nouvelles. Où par exemple le "roussi " signifie bien "grillé par la violence du feu" mais aussi les yeux "rougis" par les «rafales montantes du sommeil ». Les paradigmes s'interpénètrent sous la poussée et la direction du trait syntagmatique. En quelques mots donc: la poésie est une opération linguistique de mise en disponibilité surveillée des signifiants - ou de production du creux sémantique - afin de créer de toutes pièces l'appel du plein, ou la signification multiple.

Noël Audet

Université du Québec à Montréal et Université de Caen

1. Structure du langage poétique, Flammarion; 1966, p. 13.

2. Ibid., p. 115.

3. Ibid., p. 217.

4. La Langue manifeste, “Action poétique ", Supplément au $n^{\circ} 63,1975$, p. 99.

5. Ibid., p. 21-22.

6. L'homme rapaillé, Montréal; Les Presses de l'Université de Montréal, 1970, p. 56.

7. Ibid., p. 52.

8. Ibid., p. 48.

9. Ibid. 